\title{
Análisis del desgaste en componentes de cargadores frontales, mediante modelos basados en elementos finitos
}

\author{
Wear analysis in front loaders parts, by using finite element models \\ Paolo Fuentes $^{1} \quad$ Jaime Villanueva ${ }^{1} \quad$ Edgar Estupiñan $^{1 *}$ \\ Recibido 06 de julio de 2017, Aceptado 08 de octubre de 2018 \\ Received: July 06 de 2017, Accepted: October 08 de 2018
}

\begin{abstract}
RESUMEN
El presente trabajo tiene como objeto principal, el poder estimar el nivel de desgaste en componentes de cargadores frontales LeTourneau 1850, utilizados frecuentemente en grandes faenas mineras de Cobre, mediante la aplicación del método de los elementos finitos. Particularmente, el estudio se enfoca en partes sometidas a desgaste, conocidas como "elementos de sacrificio", durante la extracción del mineral en la roca. Cuando la falla de uno de estos elementos no es detectada previamente, bien sea durante una inspección programada, o durante la operación del equipo, y su reemplazo no se hace de manera oportuna, es posible que el daño afecte directamente la estructura del balde. De esta manera, los costos y tiempos de reparación pueden verse incrementados significativamente. Los resultados obtenidos muestran que mediante el uso de elementos finitos, es posible establecer comparativamente como cambia la concentración de cargas, identificando puntos críticos en las principales partes sometidas a desgaste.

Palabras clave: Elementos de desgaste, cargador frontal, elementos finitos.

ABSTRACT

This study uses the application of finite element method in order to estimate the level of wear in elements of front loaders LeTourneau 1850, operating in mine Doña Ines de Collahuasi. Particularly the focus of the study using finite element method is on the wear parts that are considered "sacrifice parts" due to the extraction of mineral in the rock. In case the failure of one of these elements is not detected in advance, during a programmed inspection, or during operation, the damage may affect directly to the structure of the loader. As a consequence, its reparation will be much more expansive than changing previously the wear element for a new one.
\end{abstract}

Keywords: Wear elements, front loader, finite elements.

1 Departamento de Ingeniería Mecánica. Universidad de Tarapacá. Avenida 18 de Septiembre \#2222. Arica, Chile. E-mails: paolo. fuentes.p@gmail.com; jvillanu@uta.cl; eestupin@uta.cl

* Autor de correspondencia 


\section{INTRODUCCIÓN}

El desarrollo y explotación de mineral de cobre en grandes faenas mineras, en su mayoría es realizado a cielo abierto. Por lo tanto, una gran cantidad de equipos de extracción y movimiento de tierra para la producción en la mina, es requerido. Dentro de este conjunto de equipos, se pueden identificar cuatro grandes grupos:

a. Palas: Extraen el mineral de la roca (máquinas estáticas).

b. Cargadores frontales: Extraen el mineral de la roca (máquinas dinámicas).

c. Camiones: Son los que transportan el mineral en la mina.

d. Equipos auxiliares: Excavadora, motoniveladora, camión lubricador, etc.

Tanto las grandes palas como cargadores frontales que se utilizan en faenas mineras, poseen los denominados "elementos de desgaste", que corresponden a aquellas piezas mecánicas que tienen contacto directo con el material durante los trabajos de extracción.

\section{ELEMENTOS DE DESGASTE}

Los elementos de desgaste corresponden a las partes que están sometidas al contacto directo con el mineral durante su extracción en la roca. Dichos elementos están afectos a un desgaste inevitable y progresivo en el tiempo, dadas las cargas y esfuerzos a los que están sometidos durante la operación de los equipos.

Cuando un elemento de desgaste o de "sacrificio" deja de cumplir su función y llega a su fin, debiese ser sustituido inmediatamente. Cuando esto no sucede, es posible que ocurran daños en componentes estructurales de los equipos, lo que generalmente conlleva mayores costos, comparado con el costo de sustituir una pieza de sacrificio.

Dentro de las revisiones rutinarias que se efectúan a estos equipos, la más frecuente y simple consiste en una inspección visual para estimar el nivel de desgaste presente.

Cuando un elemento tiene desgaste considerable, se propone su cambio en un próximo mantenimiento. Esto sucede para todos los elementos que presenten fisuras, grietas o que al medirlos se compruebe que su medida está por debajo del mínimo establecido para su recambio. En la Figura 1, se muestra el labio superior de un balde de cargador frontal, donde van instalados todos los elementos de desgaste.

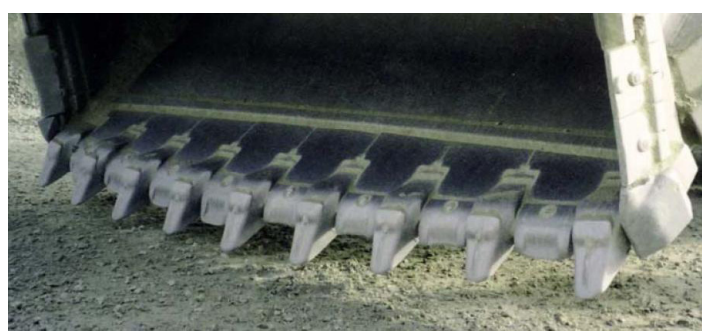

Figura 1. Balde y elementos de desgaste de cargador frontal L-1850.

Según estándares de mantenimiento establecidos, por ejemplo por fabricantes conocidos (e.g., Berkeley Forge), cuando un diente sometido a desgaste llega a su medida mínima de 9 pulgadas, éste debiese ser reemplazado por uno nuevo. Además, el fabricante sugiere cambiar todos los dientes del balde, y no solamente para algunas posiciones determinadas. Por lo tanto, esta acción correctiva debe ser planificada ya que el cambio de dientes, requiere la detención del equipo.

\section{DETECCIÓN DE DESGASTE}

La detección y estimación del desgaste en los elementos del balde, se realiza mediante dos formas de control durante las inspecciones. Primero se miden cada una de las piezas (en pulgadas), y luego se buscan fisuras en caso que haya propagación de grietas.

Es importante poder identificar los elementos críticos de desgaste, respecto de otros de menor criticidad. En el primer grupo se encuentran los dientes y entredientes. Cualquier inspección que determine un elemento caído de este grupo, detendrá completamente la operación de la maquina hasta que se logre el recambio del elemento.

A. Desgaste en dientes.

Debido a su fuerza de penetración, el desgaste en dientes ocurre por disminución de su largo [3], según se ilustra en Figura 2. Cuando la pieza alcanza su medida mínima de 9 pulg., se planifica y ejecuta el cambio del elemento. 

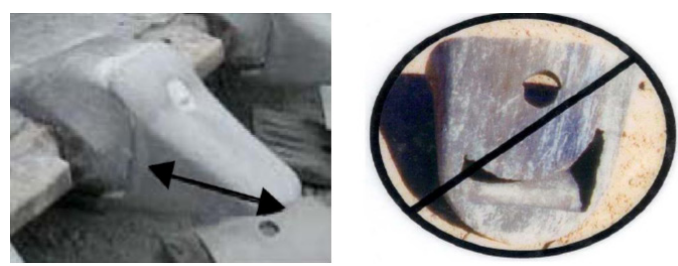

Figura 2. Desgaste en dientes.

B. Desgaste en entre-dientes.

El desgaste en entre-dientes se propaga por la curvatura del labio. Cuando se comienza a formar, es un indicador de futuras perforaciones en su zona interior, como se ilustra en la Figura 3. Si es muy pronunciada ocurre el corte del elemento durante los trabajos.

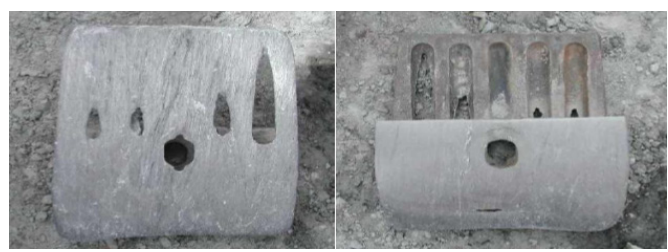

Figura 3. Desgaste en entredientes.

C. Desgaste en runners.

El desgaste en runners tiende a ser homogéneo, por disminución de su espesor, sin contar con una medida mínima al cual llegar, antes de realizar su recambio (Figura 4). Los runners pierden su condición de adhesión a la placa base del balde, en la medida que aumenta el desgaste, hasta que finalmente caen.

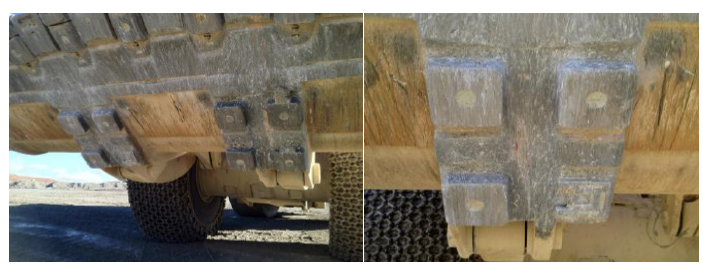

Figura 4. Desgaste en runners.

D. Desgaste en esquineros.

El desgaste en los esquineros ocurre en su zona inferior y en su curvatura (Figura 5). Cuando el desgaste es muy pronunciado en la curvatura del esquinero (E), tiende a afectar el adapter (A), y a su vez puede ocasionar también daño al diente (D).

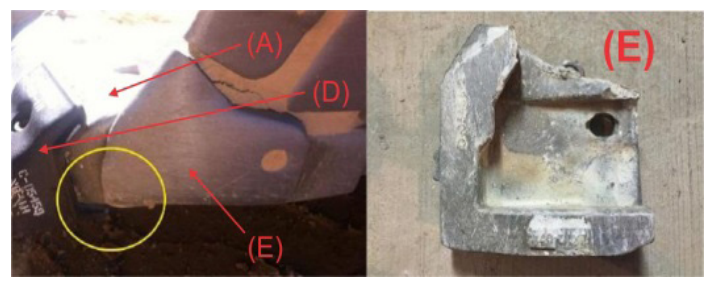

Figura 5. Desgaste en esquineros.

E. Desgaste en planchas.

El desgaste en las planchas es difícil de notar mediante inspección visual, debido a que el balde casi siempre se encuentra con carga. Generalmente se detecta cuando ya el desgaste es severo y se debe planificar una reparación (ver Figura 6).

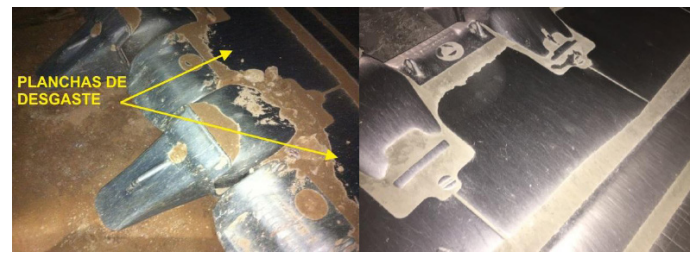

Figura 6. Desgaste en planchas.

F. Desgaste en laterales.

El desgaste de los laterales se mide respecto de su ancho. Cuando están nuevos, su ancho es cercano a 15 pulg., y cuando alcanzan 8 pulg., se debe programar su cambio. Existen diferencias entre los laterales superiores e inferiores, pero es únicamente dada su posición, según se aprecia en la Figura 7.

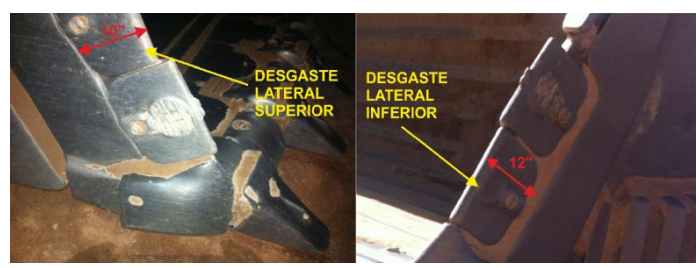

Figura 7. Desgaste en laterales.

\section{MATERIAL DE COMPONENTES}

El material de los componentes del balde corresponde a las características de un acero T-500, el cual es altamente resistente a la abrasión. Es un material muy utilizado en equipos de extracción como, excavadoras, tolvas de camiones mineros y cargadores frontales. 
Este tipo de materiales suelen contener molibdeno y silicio, y en mayor proporción manganeso y cromo. El comportamiento del cromo-manganeso, permite contar con una buena combinación de resistencia y dureza uniforme; lo cual le otorga una mejor formabilidad al acero. De esta manera, cuando el material está sometido a altas cargas de trabajo, éste se endurece a medida que las dislocaciones internas se van desplazando. Las características para el acero T-500 se muestran en las Tablas 1 y 2 (Los valores son aproximados a la composición del metal).

Tabla 1. Composición química T-500

\begin{tabular}{|c|c|}
\hline \multicolumn{2}{|c|}{ Composición química T-500 } \\
\hline C $0.22 / 0.28 \%$ & Ni $0.70 / 0.80 \%$ \\
\hline Mn $1.00 / 1.40 \%$ & Cr $1.00 / 1.30 \%$ \\
\hline Si $0.50 / 0.70 \%$ & Mo $0.30 / 0.50 \%$ \\
\hline
\end{tabular}

Tabla 2. Propiedades mecánicas T-500

\begin{tabular}{|c|c|c|c|}
\hline Acero & $\begin{array}{c}\text { Resistencia } \\
\text { tracción } \\
{\left[\mathrm{N} / \mathrm{m}^{2}\right]}\end{array}$ & $\begin{array}{c}\text { Límite } \\
\text { elástico } \\
{\left[\mathrm{N} / \mathrm{m}^{2}\right]}\end{array}$ & $\begin{array}{c}\text { Dureza } \\
\text { Brinell } \\
\mathrm{HB}\end{array}$ \\
\hline $\mathrm{T}-500$ & $3,0 \cdot 10^{11}$ & $2,05 \cdot 10^{11}$ & 500 \\
\hline
\end{tabular}

\section{ANÁLISIS MEDIANTE APLICACIÓN DE ELEMENTOS FINITOS}

En este trabajo, el análisis mediante el uso de elementos finitos, tiene como objetivo principal poder encontrar una solución al comportamiento de elementos de desgaste, cuyo modelo numérico es complejo de resolver por medios analíticos.

Para ello, el primer paso consistirá en el enmallado, para lo cual se utiliza Fortran, y será abordado con un modelo en planta 2D [2]. Posteriormente con los datos obtenidos de los esfuerzos, será posible construir un modelo 3D en SolidWorks (SW), dado que este programa permite detectar las tensiones más críticas por sobre esfuerzo.

\section{MODELAMIENTO DE LAS PIEZAS}

La construcción del modelo para las piezas se obtiene por las coordenadas de nodos establecidos para cada elemento de desgaste, esto según las medidas de las piezas. Este análisis se desarrolla por un código de programación en Fortran, cuyos datos para el enmallado son:

- Elemento cuadrilátero, 9 nodos.

- Módulo de elasticidad del acero:

$$
\mathrm{E}_{\text {young }}=2,06 \cdot 10^{11}\left[\mathrm{~N} / \mathrm{m}^{2}\right]
$$

- Coeficiente de Poisson: $v=0,3$

- Enmallado total: 44 elementos y 227 nodos.

Para la modelación en SolidWorks, se utiliza un análisis similar, y se consideran las propiedades del acero T-500, según Tabla 2. Las fuerzas aplicadas serán sometidas en un análisis vectorial de las cargas para mostrar las zonas de fractura de los elementos.

\section{ESFUERZOS POR ELEMENTO}

A continuación, se presentan las ecuaciones (1) a (19), para determinar las diferentes fuerzas y esfuerzos a los que están sometidos los elementos de desgaste. Cada una de ellas fue considerada dentro de la simulación del modelo, para estudiar el comportamiento de la deformación de las piezas en el enmallado.

i) Aceleración promedio del balde.

$$
a=\frac{|\Delta v|}{|\Delta T|}=\frac{\left|v_{2}-v_{1}\right|}{\left|t_{2}-t_{1}\right|} \cong 0,62\left[\frac{m}{s^{2}}\right]
$$

ii) Fuerzas aplicadas.

$$
\begin{aligned}
& F_{\text {diente }}=m \cdot a \approx 60[\mathrm{~N}] \\
& F_{\text {adapter }}=m \cdot a \approx 90[\mathrm{~N}] \\
& F_{\text {entrediente }}=m \cdot a \approx 80[\mathrm{~N}] \\
& F_{\text {plancha }}=m \cdot a \approx 10[\mathrm{~N}] \\
& F_{\text {lateral }}=m \cdot a \approx 30[\mathrm{~N}] \\
& F_{\text {esquinero }}=m \cdot a \approx 90[\mathrm{~N}] \\
& F_{\text {runner }}=m \cdot a \approx 40[\mathrm{~N}]
\end{aligned}
$$


iii) Esfuerzos de compresión.

$$
\begin{aligned}
& \sigma_{\text {diente }}=\frac{F}{A} \approx 2000[\mathrm{~Pa}] \\
& \sigma_{\text {adapter }}=\frac{F}{A} \approx 2500[\mathrm{~Pa}] \\
& \sigma_{\text {entrediente }}=\frac{F}{A} \approx 2000[\mathrm{~Pa}] \\
& \sigma_{\text {plancha }}=\frac{F}{A} \approx 1500[\mathrm{~Pa}] \\
& \sigma_{\text {lateral }}=\frac{F}{A} \approx 500[\mathrm{~Pa}] \\
& \sigma_{\text {esquin. }}=\frac{F}{A} \approx 1500[\mathrm{~Pa}] \\
& \sigma_{\text {runner }}=\frac{F}{A} \approx 800[\mathrm{~Pa}]
\end{aligned}
$$

iv) Esfuerzos de roce en runner y plancha.

$$
\begin{aligned}
& P_{\text {runner }}=m \cdot g \approx 600[N] \\
& F_{r}=\mu \cdot N \approx 180[N] \\
& P_{\text {plancha }}=m \cdot g \approx 180[N] \\
& F_{r}=\mu \cdot N \approx 60[N]
\end{aligned}
$$

\section{ESFUERZO DEL BALDE}

Para comprender de mejor forma el esfuerzo del balde, este debe ser evaluado en su totalidad, es decir, "como conjunto balde". Si bien el análisis por separado de las piezas para encontrar los esfuerzos sometidos de cada elemento es de ayuda para comprender el comportamiento interno; en el caso de la deformación, es necesario considerar a todas las piezas cuya deformación traiga como consecuencia la deformación de otra, y así sucesivamente.

- Carga máxima del balde:

- Área de empuje: 1 ,
Peso total de los elementos de desgaste:

$$
\begin{aligned}
& \mathrm{P}_{\mathrm{t}}=\left(9 \cdot \mathrm{P}_{\mathrm{d}}\right)+\left(9 \cdot \mathrm{P}_{\mathrm{a}}\right)+\left(8 \cdot \mathrm{P}_{\mathrm{e}}\right)+\left(8 \cdot \mathrm{P}_{\mathrm{p}}\right) \\
& +\left(4 \cdot \mathrm{P}_{\mathrm{l}}\right)+\left(8 \cdot \mathrm{P}_{\mathrm{esq}}\right)+\left(17 \cdot \mathrm{P}_{\mathrm{r}}\right) \\
& \mathrm{P}_{\mathrm{t}} \approx 5000[\mathrm{~kg}]
\end{aligned}
$$

Dónde:

$\mathrm{P}_{\mathrm{d}}$ : Peso de los dientes

$\mathrm{P}_{\mathrm{a}} \quad$ : Peso de los adapter

$\mathrm{P}_{\mathrm{e}}$ : Peso de los entredientes

$\mathrm{P}_{\mathrm{p}} \quad$ : Peso de las planchas

$\mathrm{P}_{1} \quad$ : Peso de los laterales

$\mathrm{P}_{\text {esq }}$ : Peso de los esquineros

$P_{r} \quad$ : Peso de los runners

Esfuerzo de compresión del balde:

$$
\sigma_{c}=\frac{P_{\text {total aplicado }}}{A_{\text {sección de empuje }}} \approx 6,47\left[\frac{\mathrm{kg}}{\mathrm{cm}^{2}}\right]
$$

El esfuerzo de compresión del balde según ecuación (20), será considerado como un esfuerzo adicional de empuje, en compresión, de los elementos de desgaste bajo condición de trabajo.

\section{MODELAMIENTO Y RESULTADOS ANÁLISIS 2D}

El modelo presentado en la Figura 8, representa la modelación en el plano 2D de los elementos sin desgaste, utilizando el enmallado en Fortran. Un segundo modelo también es analizado para los elementos con desgaste [4]. Entre ambos se realiza la comparación de desgaste y la influencia de la deformación.

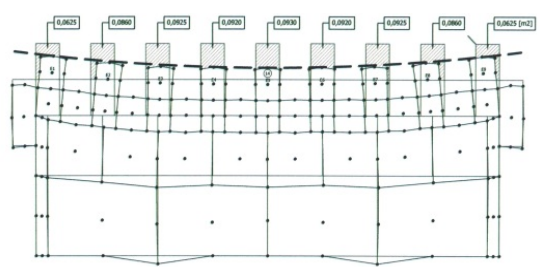

Figura 9. Deformación área desplazada, para dientes en 17 ". 


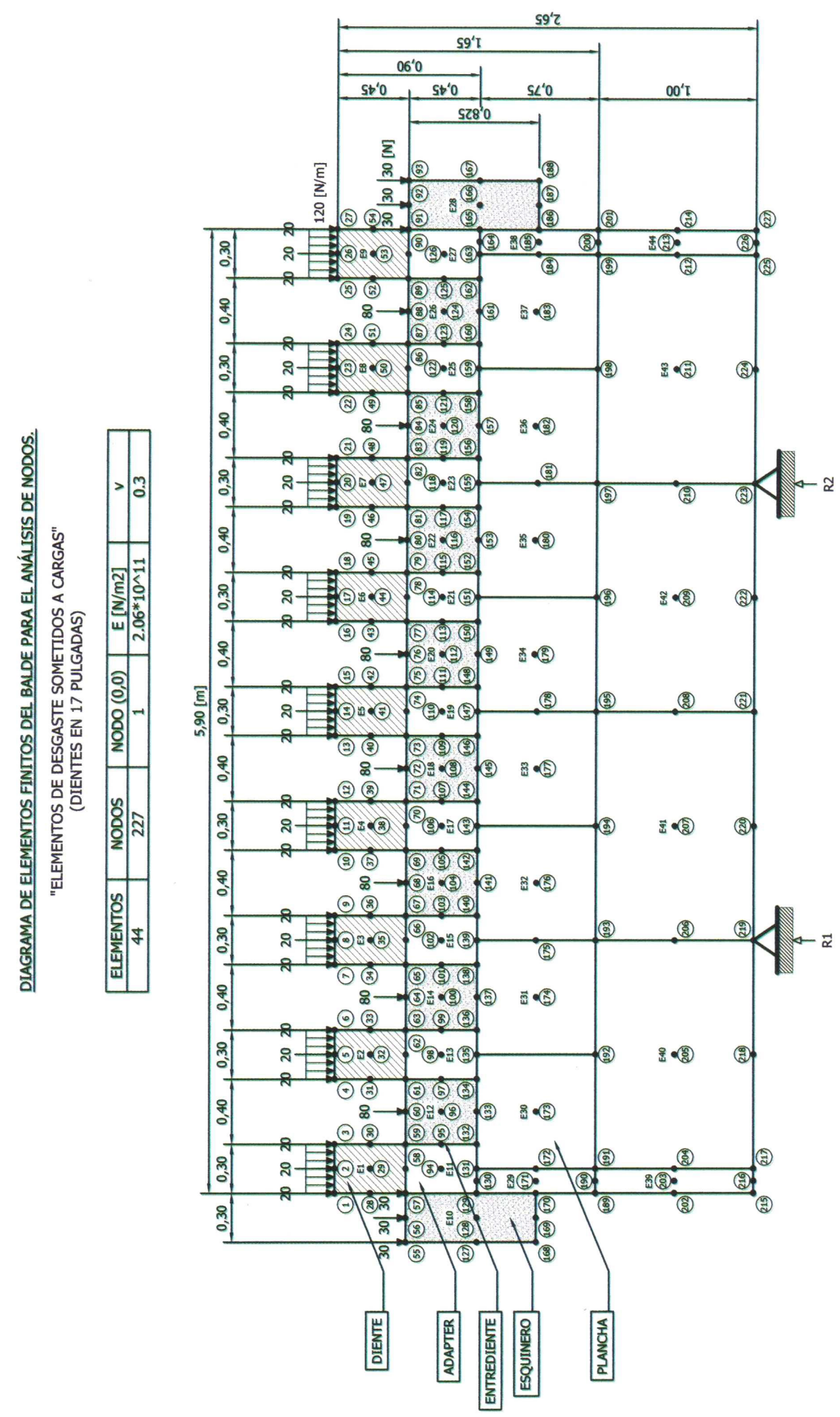

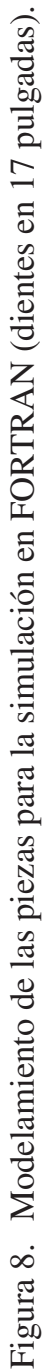




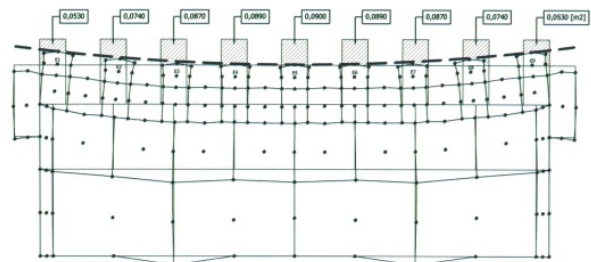

Figura 10. Deformación área desplazada, para dientes en 9".

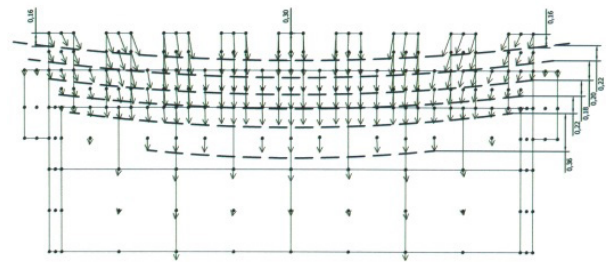

Figura 11. Concentración esfuerzos internos, para dientes en 17 ".

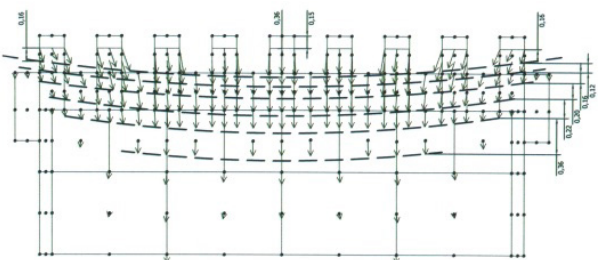

Figura 12. Concentración esfuerzos internos, para dientes en 9".

En las Figuras 9 y 10, se muestra el desplazamiento del área producto de la deformación de los elementos de desgaste sometidos a cargas de trabajo. En comparación a los resultados obtenidos para dientes en 17" y 9", se nota una tendencia de la deformación hacia una curva que se hace menos pronunciada a medida que los dientes van haciéndose más cortos producto del desgaste. Esto ocasiona que los extremos sean las zonas con mayor pérdida de material, ya que el elemento tiende a mantenerse rígido, no así en su centro donde la deformación causa que el desgaste sea menos severo.

Tabla 3. Relación desplazamiento de área en dientes 17 " y 9 "

\begin{tabular}{|c|c|c|c|}
\hline $\mathrm{N}^{\circ}$ & $\begin{array}{c}\mathrm{A}_{17^{\prime \prime}} \\
{\left[\mathrm{m}^{2}\right]}\end{array}$ & $\begin{array}{c}\mathrm{A}_{9{ }^{\prime \prime}} \\
{\left[\mathrm{m}^{2}\right]}\end{array}$ & $\begin{array}{c}\text { Desplazamiento } \\
\mathrm{A}_{17^{\prime \prime}}-\mathrm{A}_{9^{\prime \prime}} \\
\mathrm{A}_{17^{\prime \prime}}\end{array} \cdot 100$ \\
\hline 1 & 0,0625 & 0,0530 & 15 \\
\hline 2 & 0,0860 & 0,0740 & 14 \\
\hline 3 & 0,0925 & 0,0870 & 6 \\
\hline 4 & 0,0920 & 0,0890 & 3,5 \\
\hline 5 & 0,0930 & 0,0900 & 3,0 \\
\hline 6 & 0,0920 & 0,0890 & 3,5 \\
\hline 7 & 0,0925 & 0,0870 & 6 \\
\hline 8 & 0,0860 & 0,0740 & 14 \\
\hline 9 & 0,0625 & 0,0530 & 15 \\
\hline
\end{tabular}

La Tabla 3 muestra la relación de desplazamientos para dientes en 17" y 9", el cual condiciona a los dientes en las posiciones 1, 2, 8 y 9 por desgaste de material severo. Por protocolo de cambio, cuando un diente alcanza su medida mínima sea cual fuere su posición, deben cambiarse todos los dientes del balde, sin embargo, si se evalúa la vida útil del elemento, sería más beneficioso considerar las puntas de los costados de un material más resistente que en su centro.

La curvatura por diseño del balde, está considerada para compensar la deformación, y que los elementos en el mejor de los casos tiendan a desgastarse en forma lineal. El diseño con una curvatura hacia afuera del cucharón, es común encontrarlo en la mayoría de los equipos de extracción en la industria minera, y no solo en cargadores frontales.

En las Figuras 11 y 12, se muestra que la distancia de deflexión de esta curvatura para dientes nuevos y desgastados oscila entre 300 y 360 mm (12 y 14 pulgadas aprox.). Esta distancia fue comprobada en las inspecciones del balde, donde efectivamente la curvatura del labio superior se encuentra entre estas medidas. Ahora bien, tomando nuevamente la criticidad cuando los dientes empiezan a alcanzar su medida mínima, en las tablas 4 y 5 se muestran las distancias donde ocurre la mayor concentración de cargas sobre los perfiles curvos de deformación. La numeración está tomada de arriba hacia abajo para una muestra de 6 cargas.

Tabla 4. Curvas esfuerzos internos, para dientes en 17 "'

\begin{tabular}{|c|c|l|}
\hline $\mathrm{N}^{\circ}$ & $\begin{array}{c}\text { Espesor de la curva } \\
\text { Dientes en 17" }\end{array}$ & \multicolumn{1}{|c|}{ Elemento(s) } \\
\hline $1-2$ & 0,22 & Diente-adapter \\
\hline $2-3$ & 0,20 & Adapter \\
\hline $3-4$ & 0,18 & Final adapter \\
\hline $4-5$ & 0,22 & Adapter-plancha \\
\hline $5-6$ & 0,36 & Plancha \\
\hline
\end{tabular}

Tabla 5. Curvas esfuerzos internos, para dientes en 9"

\begin{tabular}{|c|c|l|}
\hline $\mathrm{N}^{\circ}$ & $\begin{array}{c}\text { Espesor de la curva } \\
\text { Dientes en 9" }\end{array}$ & \multicolumn{1}{|c|}{ Elemento(s) } \\
\hline $1-2$ & 0,12 & Diente-adapter \\
\hline $2-3$ & 0,16 & Diente-adapter \\
\hline $3-4$ & 0,20 & Final adapter \\
\hline $4-5$ & 0,22 & Plancha \\
\hline $5-6$ & 0,36 & Plancha \\
\hline
\end{tabular}


La Tabla 4 indica que la mayor concentración de esfuerzos para dientes nuevos ocurre en la parte final del adapter, específicamente en el pasador de sujeción. Este pasador no solo sostiene a los adapters en el balde, sino que también es compartido por las planchas de desgaste.

En la medida que el diente vaya perdiendo material, las cargas son concentradas en la unión diente-adapter. Por tanto, si el elemento continúa operando, puede conllevar a una fracturar y ocasionar daños al adapter, debido a las sobrecargas de trabajo.

De esta manera, se puede establecer que a pesar de considerar crítico por desgaste severo los dientes en las posiciones 1,2, 8, 9; estos no presentan una concentración alta de cargas cuando alcanzan las 9", y por tanto, no serían elementos determinantes para ser reemplazados.

La mayor criticidad ocurre en los elementos centrales 4 , 5 y 6 , donde a pesar de encontrarse con las dimensiones permitidas (10", o incluso 11"), dichos elementos presentan criticidad por fractura.

\section{ANÁLISIS 3D}

Este análisis es complementario al realizado para el estudio de esfuerzo plano, con Fortran, y para ello se utiliza SolidWorks. Particularmente lo que se busca es determinar las zonas de criticidad producto del desgaste de los elementos en el balde. En este caso, el estudio se enfocó en siete elementos principales: dientes, adapters, entredientes, planchas, laterales, esquineros y runners. Quedan fuera de este estudio los deflectores ya que no son considerados elementos habituales de recambio al ser piezas soldadas. Sin embargo serán considerados en un análisis posterior, únicamente para el balde.

Para la modelación y el análisis en 3D, se determinaron las deformaciones de los elementos, bajo la acción de las cargas de trabajo sobre cada uno de los elementos, de manera similar a como se realizó para el análisis por esfuerzo plano [5-6]. En este caso, los resultados son obtenidos a partir de la simulación por esfuerzos de VONMISES en análisis de URES, el cual indica donde se encuentra el mayor desplazamiento de los nodos, producto de la deformación, por acción de las cargas de trabajo [7].

Dientes. Las tensiones en dientes nuevos, tienden a deformar el material en forma cóncava ocasionando que el mayor desplazamiento de nodos se presente en las puntas, como se muestra en la Figura 13. Cuando los dientes están desgastados y cerca de alcanzar su medida mínima de cambio, aumenta la criticidad que existe en la unión diente-adapter.

- Tensión máxima:

$$
4,5 \cdot 10^{4}: 1,0 \cdot 10^{6}\left[\mathrm{~N} / \mathrm{m}^{2}\right]
$$

- Deformación unitaria:

$$
8,4 \cdot 10^{-8}: 3,2 \cdot 10^{-6}\left[\mathrm{~N} / \mathrm{m}^{2}\right]
$$

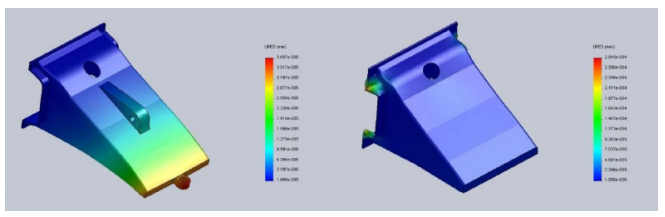

Figura 13. Resultado tensión URES para dientes.

Adapters. Según se muestra en los resultados encontrados para los adapters (Figura 14), los nodos desplazados son más críticos en la unión dienteadapter. Por tanto, para evaluar la condición de desgaste en los adapters, se recomienda revisar el estado de los alojamientos en los pasadores, además de verificar existe algún indicio de fractura.

- Tensión máxima:

$$
1,1 \cdot 10^{5}: 1,2 \cdot 10^{5} \quad\left[\mathrm{~N} / \mathrm{m}^{2}\right]
$$

- Deformación unitaria:

$$
2,1 \cdot 10^{-7}: 2,2 \cdot 10^{-7} \quad\left[\mathrm{~N} / \mathrm{m}^{2}\right]
$$

Entredientes. El desgaste en entredientes suele ser lineal cuando la pieza se encuentra en sus primeras horas de trabajo. A medida que el desgaste aumenta, la zona de criticidad se presenta en la curvatura, mostrándose cada vez más pronunciada. En la Figura 15, se muestra cómo cambia el desplazamiento en la criticidad por fractura cuando el elemento pierde material.

- Tensión máxima:

$$
6,8 \cdot 10^{4}: 2,1 \cdot 10^{5}\left[\mathrm{~N} / \mathrm{m}^{2}\right]
$$

- Deformación unitaria:

$$
2,3 \cdot 10^{-7}: 7,6 \cdot 10^{-7}\left[\mathrm{~N} / \mathrm{m}^{2}\right]
$$

Planchas. Los resultados encontrados para las planchas, muestran que existe una deformación pequeña en los bordes de las planchas (Figura 16). Los bordes son conexos con los entredientes, no obstante, a pesar de que en las planchas puedan aumentar los sobreesfuerzos por desgaste de los entredientes, la mayor criticidad en planchas se da por su desprendimiento (caída) y no por reducción de su espesor. 
- Tensión máxima:

$$
1,5 \cdot 10^{3}: 3,0 \cdot 10^{3} \quad\left[\mathrm{~N} / \mathrm{m}^{2}\right]
$$

- Deformación unitaria:

$$
3,6 \cdot 10^{-9}: 7,2 \cdot 10^{-9} \quad\left[\mathrm{~N} / \mathrm{m}^{2}\right]
$$

Laterales. Según los resultados encontrados para los laterales (Figura 17), se puede observar que la deformación tanto para elementos nuevos como con desgaste no es significativa. En los laterales, se puede considerar que el desgaste avanza de manera casi lineal. En una primera fase, cuando el elemento esta nuevo, la mayor tensión ocurre en la zona central, para luego, en una etapa más avanzada, dar paso a un desgaste casi homogéneo de la pieza.

- Tensión máxima:

$$
6,0 \cdot 10^{3}: 1,8 \cdot 10^{4} \quad\left[\mathrm{~N} / \mathrm{m}^{2}\right]
$$

- Deformación unitaria:

$$
2,0 \cdot 10^{-8}: 6,6 \cdot 10^{-8} \quad\left[\mathrm{~N} / \mathrm{m}^{2}\right]
$$

Esquineros. Según los resultados encontrados para los esquineros (Figura 18), los desplazamientos de los nodos se concentran bajo los puntos inferiores, y a medida que el elemento se va desgastando, la criticidad se traslada a la zona de alojamiento de los pasadores, ocasionando que el juego aumente. Una posible falla por fractura se puede presentar, por una combinación entre la holgura (juego) y el desgaste.

- Tensión máxima:

$$
8,0 \cdot 10^{3}: 8,5 \cdot 10^{4} \quad\left[\mathrm{~N} / \mathrm{m}^{2}\right]
$$

- Deformación unitaria:

$$
2,1 \cdot 10^{-8}: 2,1 \cdot 10^{-7} \quad\left[\mathrm{~N} / \mathrm{m}^{2}\right]
$$

Runners. Según se muestra en la Figura 19, la alta concentración de cargas en los runners, tiende a deformar el orificio central del elemento, produciendo huelgo. Posteriormente cuando el desgaste aumenta, las tensiones mayores son trasladadas a los bordes de la placa.

- Tensión máxima:

$$
6,0 \cdot 10^{3}: 1,8 \cdot 10^{4} \quad\left[\mathrm{~N} / \mathrm{m}^{2}\right]
$$

- Deformación unitaria:

$$
2,0 \cdot 10^{-8}: 6,6 \cdot 10^{-8} \quad\left[\mathrm{~N} / \mathrm{m}^{2}\right]
$$

\section{ANÁLISIS ESTRUCTURAL DEL BALDE}

El objetivo de analizar el comportamiento estructural del balde, es encontrar información sobre lo que ocurre en las zonas internas ocultas del balde cuando las piezas son deformadas producto de las

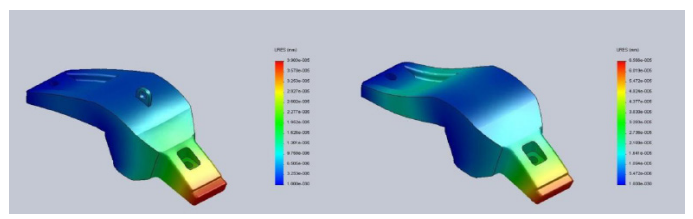

Figura 14. Simulación adapter. Resultado tensión URES.

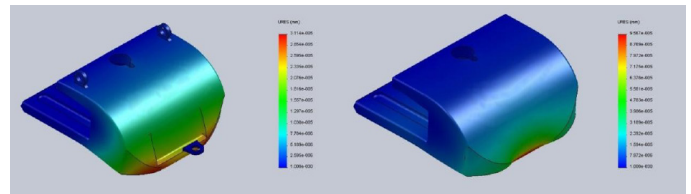

Figura 15. Simulación entrediente. Resultado tensión URES.

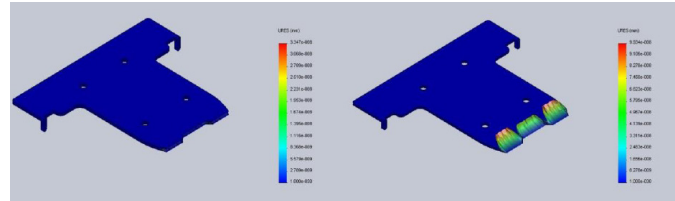

Figura 16. Simulación plancha. Resultado tensión URES.

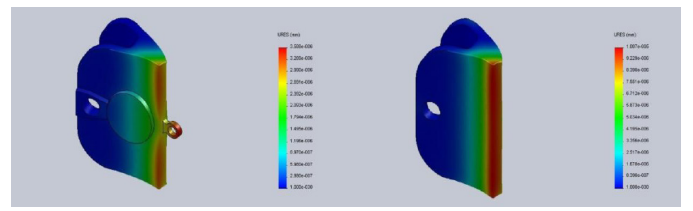

Figura 17. Simulación lateral. Resultado tensión URES.

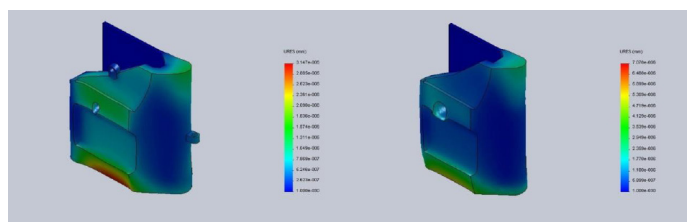

Figura 18. Simulación esquinero. Resultado tensiónURES.

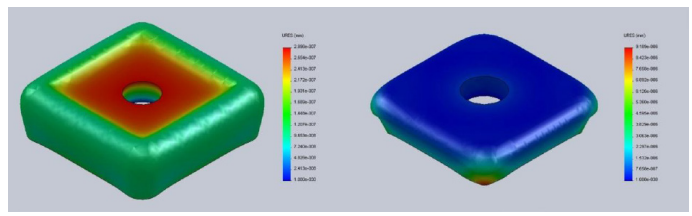

Figura 19. Simulación lateral. Resultado tensión URES.

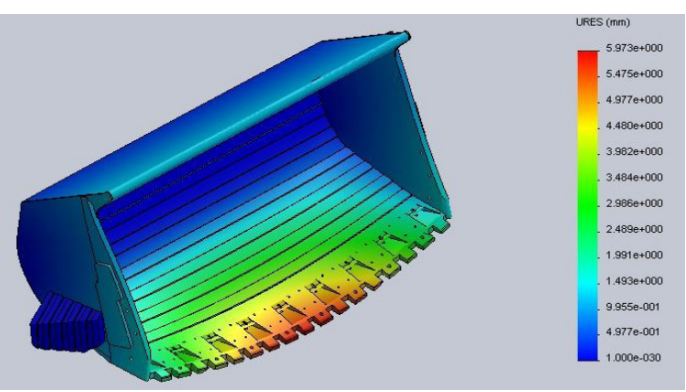

Figura 20. Simulación del balde. Resultado tensión URES. 
cargas de trabajo. Para este análisis fue necesario desmontar los elementos para poder visualizar el comportamiento del labio donde van instalados.

En ocasiones, cuando alguno de los elementos no se encuentra en su posición, bien sea por caída, como sucede usualmente, o por alguna otra razón, se produce daño en la estructura de montaje del balde. Por ejemplo, en el caso de los runners, cuando estos caen, la pérdida del elemento no solo genera el que haya una mayor deformación de la placa base, sino que también, contribuirá a la propagación de fisuras en el balde, causando daño estructural.

Los resultados de las tensiones (Figura 20), muestran que producto del empuje del balde hacia la roca, se crea en la estructura del balde esfuerzos internos en la zona central y partes laterales. Estos esfuerzos ocasionan un efecto de apertura de la boca del balde, generando compresión en los elementos centrales y tensión en las partes laterales.

\section{CONCLUSIONES}

Mediante el presente trabajo, se ha demostrado que es posible realizar un estudio comparativo del desgaste en el balde de un cargador frontal, y de los elementos que lo componen, mediante aplicaciones que hacen uso del método de los elementos finitos. Para ello se requiere contar con un registro previo de caracterización de los modos de fallo más frecuentes. De esta manera, es posible realizar análisis dimensional comparativo del desgaste, a partir de un modelo 2D, como también 3D, respecto de las partes cuando están en buen estado.

Los resultados obtenidos muestran como cambia la concentración de cargas en los elementos con desgaste. Se encontró que la deflexión máxima que puede alcanzar la deformación del labio en el balde es de $360 \mathrm{~mm}$ (entre 13 y 14 pulgadas). Para este mismo caso, la pronunciación de su curvatura debe estar entre estas medidas para asegurar que el desgaste avance de manera más homogénea entre los elementos. Esto se puede evidenciar mediante el mayor desgaste en los dientes ubicados en las esquinas del balde, respecto de la zona central. Cuando el desgaste es inicial la concentración de cargas es soportada por los adapters. A medida que los elementos empiezan a alcanzar su medida mínima, es la unión diente-adapter la que sufre mayor criticidad.

Se ha demostrado que es posible realizar una medición del desgaste comparativo entre los elementos nuevos y con desgaste. Mediante el estudio se pudo constatar el cambio de dirección de las cargas cuando los elementos pierden material, dirigiendo la concentración de esfuerzos hacia otras partes de los elementos, lo que contribuye a acelerar el final de su vida útil, provocando fractura en dichas zonas. Se evidencio también que la mayor concentración de cargas en el balde ocurre en la zona central, encontrándose que la zona inferior del balde es crítica, ya que se producen mayores tensiones por estiramiento en las vigas metálicas.

\section{REFERENCIAS}

[1] Ferdinan P. Beer. "Mechanics of Materials". McGraw-Hill, Fifth Edition. 2009.

[2] I.M. Smith. "Programming the finite element method". Offices, Fourth Edition. 2004.

[3] CAD CAE Ingeniería LTDA. "Análisis de la integridad estructural del cargador frontal CAT 994F, Mediante el método de los elementos finitos". Compañía Minera Escondida. Antofagasta-Chile. 2010.

[4] A. Adibi-Sedeh, M. Marizi, V. Pednekar and V. Madhavan. "Investigation of the effect of using different material models on finite element simulations of machining". Proceedings of the International Workshop Modelling of Machining Operations, Antalya, Turkey. August 2005.

[5] A. Camacho. "Análisis por método de los elementos finitos de procesos estacionarios de conformado por deformación plástica". Tesis Doctoral UNED. Escuela Superior de Ingeniería Industrial, Departamento de Construcción y Fabricación, España, 2005.

[6] Ceretti E., Lazzaroni C., Menegardo L. and Altan T. "Turning simulations using a threedimensional FEM code". Journal of Material Processing Technology. 2000. Vol. 98, pp. 99-103.

[7] M. Cockcroft, D. Latham. "Ductility and the workability of metals". Journal of the Institute of Metals. Vol. 96, pp. 33-39. 1968. 